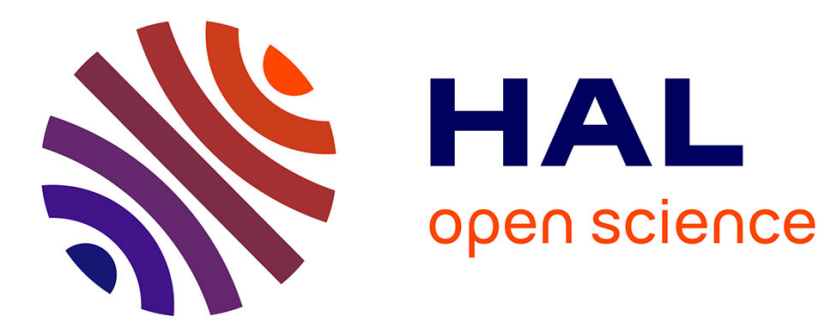

\title{
Time-resolved temperature rise in a thin liquid film due to laser absorption
}

Maria Luisa Cordero, Emilie Verneuil, François Gallaire, Charles N. Baroud

\section{To cite this version:}

Maria Luisa Cordero, Emilie Verneuil, François Gallaire, Charles N. Baroud. Time-resolved temperature rise in a thin liquid film due to laser absorption. Physical Review E : Statistical, Nonlinear, and Soft Matter Physics, 2009, 79 (1), pp.011201. 10.1103/physreve.79.011201 . hal-01002603

\section{HAL Id: hal-01002603 \\ https://hal-polytechnique.archives-ouvertes.fr/hal-01002603}

Submitted on 7 Jul 2014

HAL is a multi-disciplinary open access archive for the deposit and dissemination of scientific research documents, whether they are published or not. The documents may come from teaching and research institutions in France or abroad, or from public or private research centers.
L'archive ouverte pluridisciplinaire HAL, est destinée au dépôt et à la diffusion de documents scientifiques de niveau recherche, publiés ou non, émanant des établissements d'enseignement et de recherche français ou étrangers, des laboratoires publics ou privés. 


\title{
Time-resolved temperature rise in a thin liquid film due to laser absorption
}

\author{
María Luisa Cordero, ${ }^{1}$ Emilie Verneuil, ${ }^{1}$ François Gallaire, ${ }^{2}$ and Charles N. Baroud ${ }^{1, *}$ \\ ${ }^{1}$ LadHyX and Department of Mechanics, Ecole Polytechnique, 91128 Palaiseau, France \\ ${ }^{2}$ Laboratoire J. A. Dieudonné, Université de Nice Sophia-Antipolis, 06108 Nice, France
}

(Received 19 August 2008; published 7 January 2009)

\begin{abstract}
The temperature increase of a thin water layer is investigated, both experimentally and numerically, when the layer is heated by an infrared laser. The laser is focused to a waist of $5.3 \mu \mathrm{m}$ inside a $28 \mu \mathrm{m}$ gap that contains fluorescent aqueous solutions between two glass slides. Temperature fields are measured using the temperature sensitivity of rhodamine- $\mathrm{B}$, while correcting for thermal diffusion using rhodamine-101, which is insensitive to temperature. In the steady state, the shape of the hot region is well fitted with a Lorentzian function whose width ranges between 15 and $30 \mu \mathrm{m}$, increasing with laser power. At the same time, the maximum temperature rise ranges between 10 and $55^{\circ} \mathrm{C}$ and can display a decrease at high laser powers. The total energy stored in the sample increases linearly with the laser power. The dynamics of the heating occurs with two distinct time scales: (i) a fast time $\left(\tau_{\Theta}=4.2 \mathrm{~ms}\right.$ in our case) which is the time taken to reach the maximum temperature at the laser position and the maximum temperature gradient, and (ii) a slow time scale for the spatial profile to reach its final width. The temperature field obtained numerically agrees quantitatively with the experiments for low laser powers but overpredicts the temperature rise while underpredicting the profile width for high powers. The total energy shows good agreement between experiments and simulations for all laser powers, suggesting that the discrepancies are due to a broadening of the laser, possibly due to a thermal lensing effect.
\end{abstract}

DOI: 10.1103/PhysRevE.79.011201

PACS number(s): 44.10. $+\mathrm{i}, 66.25 .+\mathrm{g}$

\section{INTRODUCTION}

Laser radiation offers a useful technique to heat an absorbing sample in a localized way. Indeed, since a laser beam can be be focused to its diffraction limit, it can be used to inject energy at scales that are difficult to reach with other techniques, without requiring any specific microfabrication such as electrode deposition. Examples of applications where such heating has been applied include the evaporation of contaminants [1], the study of Marangoni flows [2], the measurement of media properties such as small absorbancies [3] and Soret coefficients [4], and the study of cell processes [5] and of material properties under high pressure and temperature conditions in diamond anvil cells [6]. However, heating can also appear as an undesirable side effect in optical manipulation studies $[7,8]$.

Recently, laser-induced heating was used to provide active control over droplet evolution in microfluidic channels, through the local manipulation of surface tension between water and oil [9]. In these studies, a laser was focused through a microscope objective onto the surface of a water drop in order to locally vary the interfacial tension and thus create a Marangoni flow. The net force due to this flow provides a way to control drop formation, routing, fusion, and division [10].

The potential usefulness of the laser heating is limited in two ways. First, it is important to quantify the dynamics of the temperature increase; even though miniaturization leads to a reduction of the thermal inertia and a shortening of the characteristic heating time, this time often needs to be compared with other short times, such as the time for the drop

\footnotetext{
*charles.baroud@ladhyx.polytechnique.fr
}

interface to pass the laser position in the example of microfluidic drop control.

Second, many applications, such as thermophoresis or thermocapillarity, depend on the temperature gradient rather than the temperature itself, making it important to know the size of the region that is heated by the laser. Indeed, although the heating is localized, the width of the hot region is expected to be larger than the size of the laser waist. Moreover, in practical applications of microfluidics or optical traps the temperature rise in the liquid can damage some biological samples or skew chemical measurements inside the microfluidic droplet.

In what follows, we concentrate on the heating of a thin liquid layer by a continuous wave laser that is focused to a small spot inside the sample. The presence of the top and bottom solid boundaries plays a fundamental role in the heat flux in our geometry, contrary to the assumptions in the existing work on the subject which addresses a localized heating in an infinite medium [3,7,11-13]. Fluorescence measurements of temperature provide spatially and temporally resolved temperature fields [14-16] and we correct for thermally induced migration by developing a method based on two different fluorophores. This allows us to explore higher temperature ranges than in previous work [17]. Finally, the experimental results are augmented by numerical solutions of the heat equation, which provide a way to explore different wall materials and layer thicknesses.

Below, we begin with a description of the experimental setup and method, followed by a theoretical treatment of the problem in Sec. III. The results of Sec. IV are divided into three parts, first describing the dynamics of the temperature rise in the transient regime, followed by the steady state spatial distribution, and finally by the results of the numerical simulation. The discussion summarizes the results and com- 


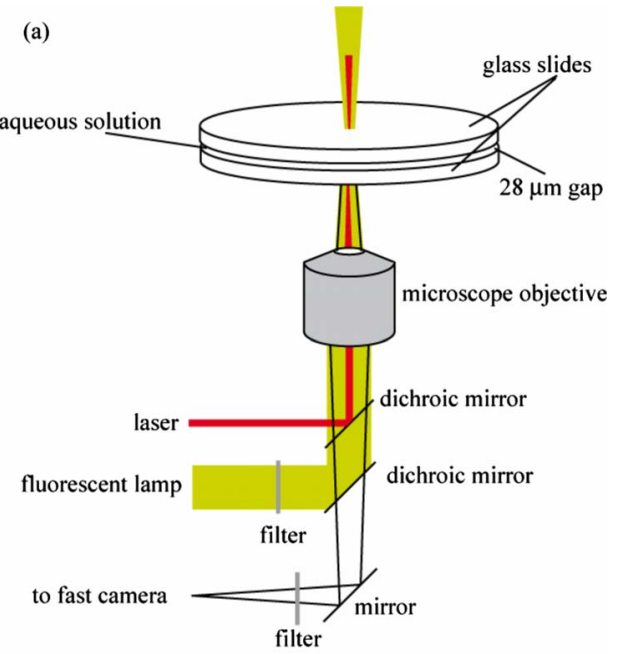

ments on their utility for predicting the temperature field in specific cases.

\section{EXPERIMENTAL SETUP}

The experimental setup consists of an inverted microscope (Nikon ECLIPSE TE2000-U) with epifluorescent illumination from a metal halide lamp (Exfo X-Cite 120), as shown in Fig. 1(a). An infrared laser beam of wavelength $\lambda=1480 \mathrm{~nm}$ is collimated to a parallel Gaussian beam whose radius is measured at $\omega_{1}=2.2 \mathrm{~mm}$. The laser is focused inside the sample through the microscope objective (Nikon, Plan Fluor $10 \times / 0.3$ ) of focal length $f=20 \mathrm{~mm}$ to a Gaussian spot whose waist can be calculated, using Gaussian optics, to be $\omega_{0}=\sqrt{n \omega_{1}^{2} /\left[1+\left(Z_{1} / f\right)^{2}\right]}$. Here, $n=1.5$ is the refractive index of glass and $Z_{1}=\pi \omega_{1}^{2} / \lambda$, which yield $\omega_{0}=5.3 \mu \mathrm{m}$. The laser power $P_{0}$ is measured at the sample position through a single glass slide and spans the range 10.5 to $132.8 \mathrm{~mW}$.

At this wavelength, part of the optical power is absorbed by the water, while the solid walls absorb almost none, as shown by the absorption coefficient $\alpha$ of Table I. This table also lists the thermal diffusivity $\chi$ and thermal conductivity $\kappa$ for the three materials that will be discussed below.

Fluorescence images are recorded using a fast camera (Photron Fastcam 1024 PCI), triggered by the same external signal as the laser, taking images at 500 frames per second. For each measurement, 50 images are recorded before the laser is switched on and averaged to be used as a roomtemperature background. The fluorescence of the heated sample is then followed by recording 500 images after the trigger signal. The imaged region is a $1.74 \times 1.74 \mathrm{~mm}$ square. The spatial resolution of our measurements (1.7 $\mu \mathrm{m} /$ pixel) is given by the microscope objective and the number of pixels on the camera sensor. The temporal resolution, on the other hand, is limited by the camera sensor's sensitivity, which dictates the maximum frame rate that can still yield sufficient contrast between the hot and cold regions. For our conditions, this time is $2 \mathrm{~ms}$.

The sample, sketched in Fig. 1(b), consists of $200 \mu \mathrm{l}$ of a fluorescent aqueous solution confined between two glass slides of diameter $76.2 \mathrm{~cm}$ and thickness $H=1 \mathrm{~mm}$. A ring of a photoresist (SU-8 2035, Microchem) of inner diameter $13 \mathrm{~mm}$ (not shown) is patterned on one of the glass slides by lithography and forms the chamber which contains the solution and prevents its evaporation. The height of the ring, $2 h=28 \mu \mathrm{m}$, was measured using a contact profilometer. Finally, a $150 \mathrm{~g}$ weight is set on the upper glass slide to squeeze out the excess solution and seal the chamber. The resulting thickness of the liquid is small compared to the absorption length of the water $(400 \mu \mathrm{m})$, so that the BeerLambert law for laser absorption can be approximated by its linearization. The thickness is also small compared to the Rayleigh zone, $L_{R}=\pi \omega_{0}^{2} / \lambda \approx 130 \mu \mathrm{m}$, which defines the distance over which the beam can be considered focused. The laser can therefore be considered divergence-free over this length.

\section{A. Fluorophore solutions}

Two different solutions are used in this study: the first is a rhodamine-B (rho-B) aqueous solution [Reactifs RAL, molecular weight $(\mathrm{MW})=479.02,50 \mathrm{mg} / 1$ in $50 \mu \mathrm{M}(4-(2-$

TABLE I. Thermal properties of the materials at room temperature. The material properties can vary with temperature.

\begin{tabular}{lccc}
\hline \hline & Diffusivity $\chi$ & Conductivity $\kappa$ & Absorption coefficient $\alpha$ \\
\hline Water & $1.3 \times 10^{-7} \mathrm{~m}^{2} / \mathrm{s}$ & $0.54 \mathrm{~W} / \mathrm{m} \mathrm{K}$ & $2354 \mathrm{~m}^{-1}$ \\
Glass & $8.5 \times 10^{-7} \mathrm{~m}^{2} / \mathrm{s}$ & $1.38 \mathrm{~W} / \mathrm{m} \mathrm{K}$ & $39.3 \mathrm{~m}^{-1}$ \\
Polydimethylsiloxane & $1.4 \times 10^{-7} \mathrm{~m}^{2} / \mathrm{s}$ & $0.18 \mathrm{~W} / \mathrm{m} \mathrm{K}$ & $14.3 \mathrm{~m}^{-1}$ \\
\hline \hline
\end{tabular}




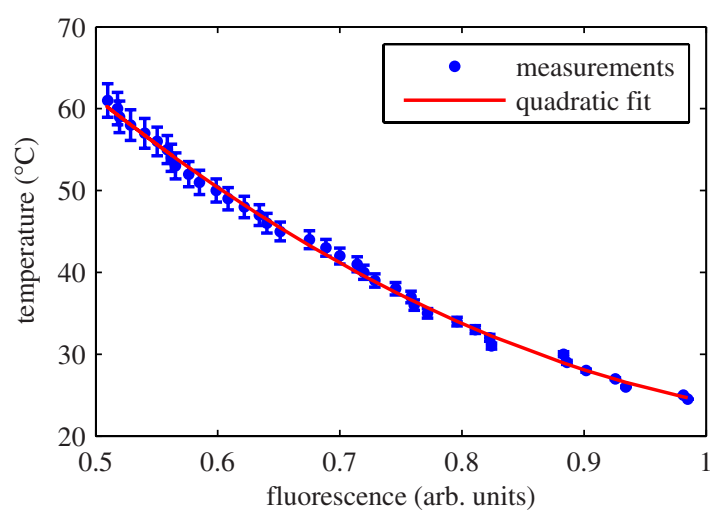

FIG. 2. (Color online) Temperature calibration of the rho-B solution fluorescence.

hydroxyethyl)-1-piperazineethanesulfonic acid) (HEPES) buffer, $p \mathrm{H}=7$ ], whose fluorescence quantum yield (emitted quanta per absorbed quanta) decreases with temperature $[18,19]$, and whose fluorescence variation can be calibrated over a wide range of temperatures [16]. The second is an aqueous solution of rhodamine-101 (rho-101) (Fluka, MW $=490.59,30 \mathrm{mg} / 1$ in $50 \mu \mathrm{M}$ HEPES buffer, $p \mathrm{H}=7$ ) whose fluorescence quantum yield is virtually independent of temperature $[14,18,20]$. The two molecules are similar and have a similar size, the main difference being the presence of diethylamino groups in the rho-B molecule, whose rotational freedom makes its quantum yield sensitive to temperature [18].

The calibration of the fluorescence dependence of the rho-B solution on temperature is done, in the absence of the laser beam, by placing a $150 \mathrm{ml}$ beaker of hot water on top of the glass slides. Thermal contact between the reservoir and the sample is provided by a layer of water, which allows us to consider that the sample temperature is the same as the beaker's. The temperature of the water reservoir is monitored as it cools down from $80^{\circ} \mathrm{C}$ to room temperature. For every $1{ }^{\circ} \mathrm{C}$ step, thirty images of the sample are taken at a frame rate of 500 frames/s and normalized by an image taken at room temperature, to correct for inhomogeneous illumination. The fluorescence dependence on temperature is obtained as the spatial and temporal mean value over the 30 images, which is then fitted with a quadratic function, as shown in Fig. 2. Note that the sample temperature is shifted to lower values with respect to the monitored temperature due to heat transfer into the surrounding air at room temperature, the shift being higher at higher temperatures. This fact is estimated using a convection heat transfer model [21] due to natural convection in the air and produces the error bars shown in Fig. 2.

\section{B. Thermophoresis correction}

When the laser is turned on, a decrease in fluorescence intensity around the laser focus is observed for both solutions. The details of the decrease, however, differ for the two dyes: In the case of rho-101, the dark central spot is surrounded by a bright ring at a radius $r \approx 50 \mu \mathrm{m}$, as shown in Fig. 3(a). At later times, the dark spot continues to become
Rhodamine 101
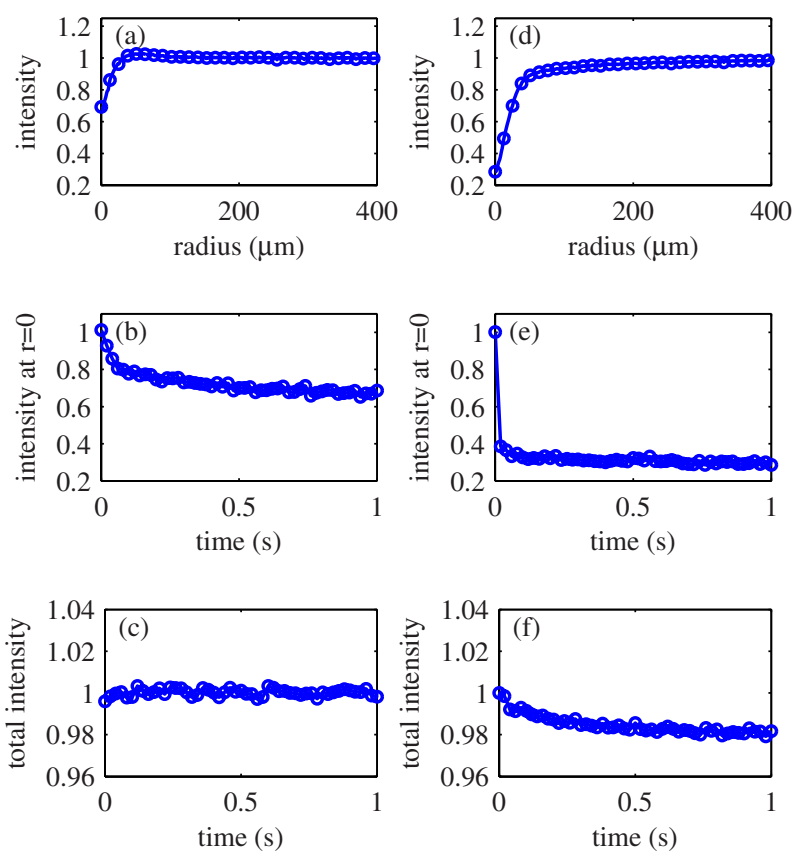

FIG. 3. (Color online) (a), (d) Radial fluorescence profiles after $1 \mathrm{~s}$ of laser heating with $P_{0}=76.4 \mathrm{~mW}$ for the rho- 101 and rho-B solutions, respectively. (b), (e) Fluorescence intensity at $r=0$ as a function of time for the rho-101 and rho-B solutions, respectively. (c), (f) Total fluorescence intensity as a function of time for the rho-101 and rho-B solutions respectively. Intensity scales are in arbitrary units.

darker even after one second of heating [Fig. 3(b)]. Simultaneously, the ring expands and becomes less bright, in such a way that the total intensity, defined as the intensity integrated over the whole image, remains constant as a function of time [Fig. 3(c)].

The azimuthal average of the rho-B solution, plotted in Fig. 3(d), displays a stronger decrease than in the former case and no evidence of a bright ring is present. Furthermore, the fluorescence at the laser location initially decreases much faster, followed by a slower evolution [Fig. 3(e)]. Finally, the total fluorescence intensity of rho-B is not conserved, as shown in Fig. 3(f), although the total fluorescence intensity returns to its initial value once the laser is switched off.

Since the rho-101 fluorescence quantum yield is not sensitive to temperature, the dark spot is solely due to a decrease in the concentration of dye molecules. This migration of molecules is due to their diffusion down the thermal gradient, an effect known as thermophoresis, thermal diffusion or Soret effect $[17,22]$. This is further confirmed by the conservation of the total intensity, indicating that molecules have been redistributed through the sample, the bright ring corresponding to a radial accumulation of the molecules repelled from the high temperature region ("hot spot"). In the case of rho-B the fluorescence decrease is in part due to the temperature dependence of its quantum yield, with thermophoresis responsible for an additional decrease in fluorescence at a slower time scale.

Since thermophoresis cannot be avoided in the presence of inhomogeneous thermal fields, the method we employ 
takes advantage of the similarity in size between the two rhodamine molecules to correct the temperature and concentration dependence in the rho-B images by the strictly concentration dependence for the rho-101. To that end, the same heating experiment is repeated using the two solutions and images are recorded with the same frame rate. The concentration variation is obtained from the rho-101 images, which is then used to normalize the rho-B image at each time step. In this way, temporally and spatially resolved temperature profiles are obtained.

\section{THEORY}

The theoretical description of the heating of a medium by absorption of a laser beam has been addressed by several authors in the past. The first analytical description was developed by Carslaw and Jaeger in 1959 [11], who solved the heat equation for an infinitely extended opaque medium, heated by a Gaussian laser beam, using a Green's function method. The result was later extended in 1965 by Gordon et al. [3] for an infinitely long cylinder. However, neither formulation takes into account the presence of top and bottom boundaries which play a major role in dissipating the heat. For this reason, the temperature increase predicted by both formulations highly exceeds our measurements.

More recent work describes the heating caused by the highly focused laser beams used in optical traps $[7,12,13]$. Again, the effects of the boundaries are neglected, this time based on the fact that the laser absorption is produced in a spherical region of the size of the laser focus. For the high numerical aperture optics of optical traps, this is typically of the order of $1 \mu \mathrm{m}$, much smaller than the size of the chamber. In our system, however, the size of the heated volume is comparable to the chamber thickness and therefore a different model is necessary to describe the laser heating.

Consider a liquid layer, of thermal conductivity $\kappa$ and thermal diffusivity $\chi$, contained between two solid walls of thermal conductivity $\kappa_{\mathrm{s}}$ and diffusivity $\chi_{\mathrm{s}}$, as sketched in Fig. 1(b). The absorbing liquid is heated by a laser beam, focused at $r=0$, of intensity $I(r)=2 P_{0} / \pi \omega_{0}^{2} \exp \left(-2 r^{2} / \omega_{0}^{2}\right)$. The total absorbed laser power is $P_{\text {in }}=2 \alpha h P_{0}$, where $\alpha$ is the absorption coefficient of the medium at the laser wavelength. The whole system is immersed in a thermal bath at room temperature. Since $a \gg \omega_{0}$ and $H \gg 2 h$, the temperature rise is negligible at the lateral boundaries, $r=a$, and at the outer limits of the walls, $z= \pm(H+h)$.

The elevation of temperature in the fluid, $T$, is described by the heat equation with a heat source term $\dot{q}$ due to the laser absorption $[3,4,11]$ :

$$
\begin{gathered}
\frac{\partial T}{\partial t}=\chi \nabla^{2} T+\frac{\chi}{\kappa} \dot{q}, \\
\dot{q} \approx \alpha I(r),
\end{gathered}
$$

completed by the boundary conditions which account for the temperature and heat flux continuity at the fluid-solid boundary:

$$
T(r, z= \pm h, t)=T_{\mathrm{s}}(r, z= \pm h, t),
$$

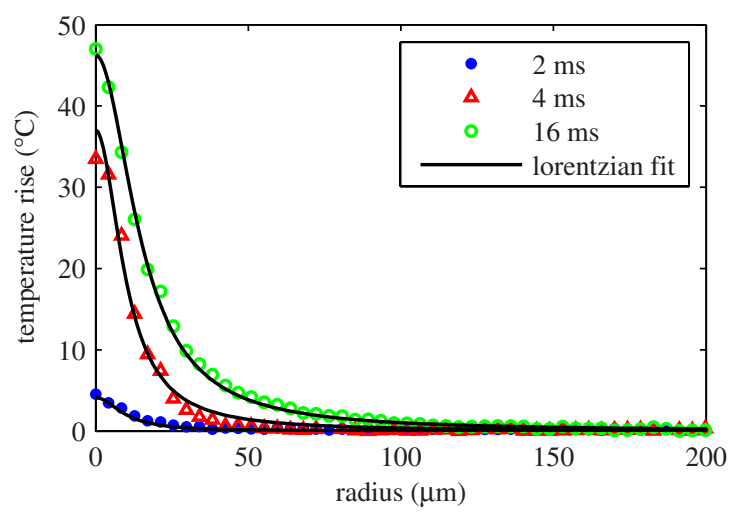

FIG. 4. (Color online) Evolution of the radial temperature profile. The laser power is $P_{0}=76.4 \mathrm{~mW}$, which corresponds to an absorbed power of $P_{\text {in }}=4.5 \mathrm{~mW}$.

$$
\kappa\left(\frac{\partial T}{\partial z}\right)_{(r, z= \pm h, t)}=\kappa_{\mathrm{s}}\left(\frac{\partial T_{\mathrm{s}}}{\partial z}\right)_{(r, z= \pm h, t)},
$$

where the subscript "s" indicates the values in the solid. The external temperature is assumed fixed. As Eqs. (3) and (4) suggest, the temperature fields in the liquid and solid are coupled and one cannot be solved without the other. This system is easiest solved using numerical simulations, as done below in Sec. IV.

\section{RESULTS}

\section{A. Dynamics of the temperature increase}

Three experimental depth-averaged temperature profiles are shown in Fig. 4 for an absorbed laser power $P_{\text {in }}$ $=4.5 \mathrm{~mW}$ and times corresponding to 2,4 , and $16 \mathrm{~ms}$ after the laser is turned on. The temperature in the fluid rises nearly $50{ }^{\circ} \mathrm{C}$ in a few milliseconds while the hot spot becomes wider. However, the temperature increase remains less than $1.7^{\circ} \mathrm{C}$ at radii larger than $200 \mu \mathrm{m}$. The experimental data were accurately fitted by a Lorentzian curve:

$$
\bar{T}(r, t)=\frac{\Theta(t)}{1+[r / \sigma(t)]^{2}},
$$

with two fitting parameters: $\Theta(t)$, which corresponds to the temperature increase at the laser location, and $\sigma(t)$, which is the half width at mid-height of the profile. This form of the temperature field agrees with those published by Duhr et al. [23], although the laser powers are much larger here.

The evolution of $\Theta(t)$ and $\sigma(t)$ is shown in Figs. 5(a) and $5(\mathrm{~b})$, respectively. $\Theta(t)$ increases rapidly, reaching its maximum value $\Theta_{\infty}$ within $10 \mathrm{~ms}$. In contrast, $\sigma(t)$ exhibits a fast increase at small times followed by a slower evolution, still slowly increasing after $1 \mathrm{~s}$ of laser heating. This increase is small, however, $\sigma(t)$ remaining around a value of $20 \mu \mathrm{m}$. For practical purposes, we will consider the steady state to be reached after $0.5 \mathrm{~s}$ of laser heating. It should be noted here that a slight difference between the thermophoretic behaviors of the two dyes may induce an error in the temperature field measurement that would be reflected in an unsteady value of $\sigma(t)$. 

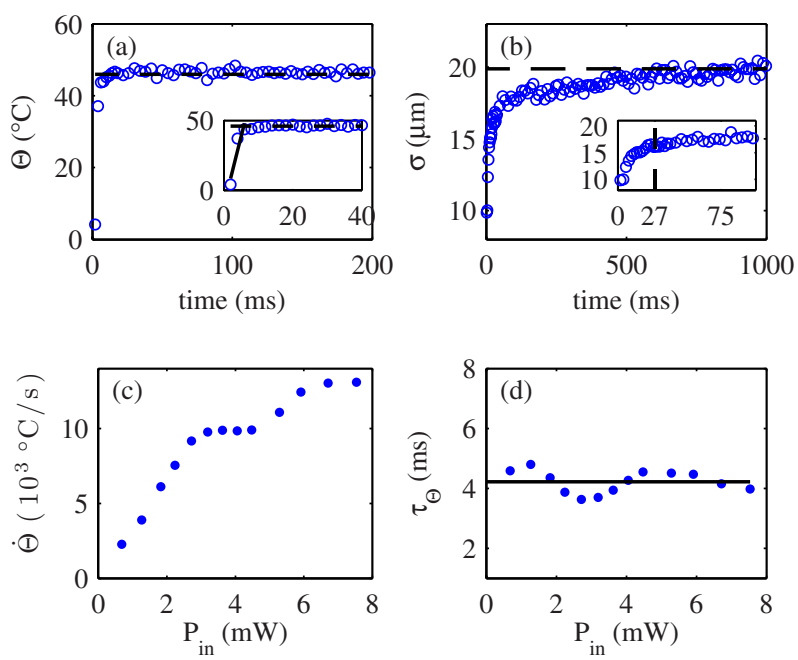

FIG. 5. (Color online) (a) $\Theta(t)$ for $P_{\text {in }}=4.5 \mathrm{~mW}$. The dashed line indicates the maximum temperature increase $\Theta_{\infty}$. In the inset the first $40 \mathrm{~ms}$ are shown, and the solid line corresponds to a linear fit of the first points. (b) $\sigma(t)$ for the same data. The dashed line indicates the value of $\sigma_{\infty}$. The inset shows the first $100 \mathrm{~ms}$. (c) $\dot{\Theta}$ as a function of $P_{\text {in }}$. (d) $\tau_{\Theta}$ as a function of $P_{\text {in }}$. The solid line indicates its mean value.

The heating rate $\dot{\Theta}$ is arbitrarily defined as the slope of a linear fit of $\Theta(t)$ for $t \leqslant 6 \mathrm{~ms}$ [see inset of Fig. 5(a)]. $\dot{\Theta}$ increases with laser power, as seen in Fig. 5(c), meaning that the rate of the temperature increase at the origin is larger for larger laser powers. Furthermore, a thermal time can be extracted from this heating rate as $\tau_{\Theta}=\Theta_{\infty} / \dot{\Theta}$. This time scale measures the time necessary to reach the maximum temperature at the laser position. It is independent of the laser power, with a mean value $\left\langle\tau_{\Theta}\right\rangle=4.2 \mathrm{~ms}$, as shown in Fig. 5(d).

Note that it is the gradient of temperature that determines thermocapillary effects. It is interesting therefore to measure the evolution of the gradient as a function of time. From the Lorentzian fit, the maximum thermal gradient occurs at a radius $\sigma(t) / \sqrt{3}$ and its value $-3 \sqrt{3} \Theta(t) / 8 \sigma(t)$ is plotted in Fig. 6. Indeed, the fast rise of the central temperature leads to a high value of the gradient at early times, which then asymptotes to its long term value as the hot spot spreads. The time to reach the maximum temperature gradient is well described by the time $\tau_{\Theta}$ for all laser powers. For $P_{\text {in }}$ $=4.5 \mathrm{~mW}$ and $t=4 \mathrm{~ms}$ we find $\sigma=10 \mu \mathrm{m}$ and $\Theta=37^{\circ} \mathrm{C}$, yielding a thermal gradient of $-2.4^{\circ} \mathrm{C} / \mu \mathrm{m}$.

\section{B. Steady state}

Next, we consider the depth-averaged temperature field at late times $\bar{T}_{\infty}(r)$, which was computed by averaging the temperature distributions in the range $0.5<t<1 \mathrm{~s}$. An example is shown in Fig. 7(a) for an absorbed laser power $P_{\text {in }}$ $=4.5 \mathrm{~mW}$. Again, the steady state radial profile is fitted by a Lorentzian curve that gives the temperature increase at the center $\Theta_{\infty}$ and width $\sigma_{\infty}$ of the hot spot as a function of $P_{\text {in }}$. Figure $7(\mathrm{~b})$ shows that the amplitude $\Theta_{\infty}$ ranges from $10{ }^{\circ} \mathrm{C}$ for $P_{\text {in }}=0.68 \mathrm{~mW}$ to $55^{\circ} \mathrm{C}$ for $P_{\text {in }}=5.9 \mathrm{~mW}$, before decreas-

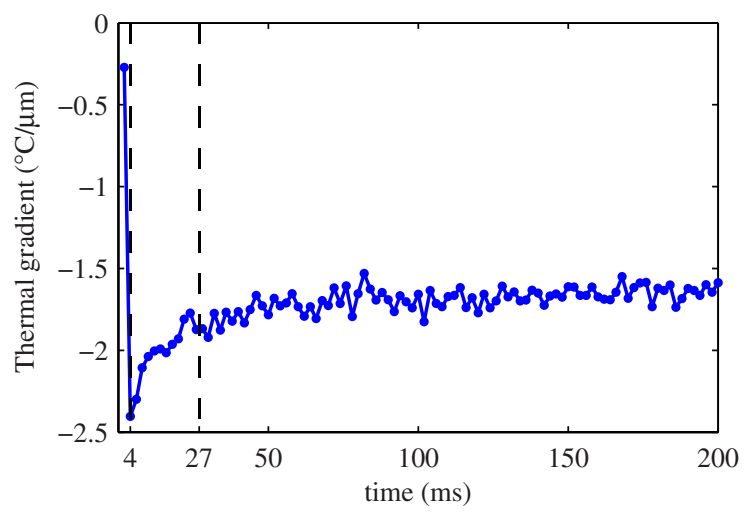

FIG. 6. (Color online) Evolution of the maximum thermal gradient after the laser is turned on, for an absorbed power $P_{\text {in }}$ $=4.5 \mathrm{~mW}$. The gradient reaches its maximum absolute value at $t$ $=4 \mathrm{~ms}$.

ing again at higher laser powers. This nonlinear behavior is accompanied by a nonmonotonic increase of the steady state width of the hot spot $\sigma_{\infty}$ as a function of $P_{\text {in }}$, as shown in Fig. $7(\mathrm{c})$.

Note that the temperatures that are measured near the laser location for $P_{\text {in }}>4 \mathrm{~mW}$ are outside the calibration range of Fig. 2. However, the values plotted in Fig. 7(b) are obtained from the fit over the whole temperature profile rather than actually observed at the spot center in the experiments. Since the profiles are well described by the Lorentzian curve, we assume that the values that are plotted correspond to the real physical temperatures.

Finally, the thermal energy $\Delta E$ stored by the sample, defined as

$$
\Delta E=2 h \frac{\kappa}{\chi} \int_{0}^{a} \bar{T}_{\infty}(r) 2 \pi r d r,
$$

was calculated by integrating the experimental temperature profiles. $\Delta E$ was found to increase linearly with the absorbed
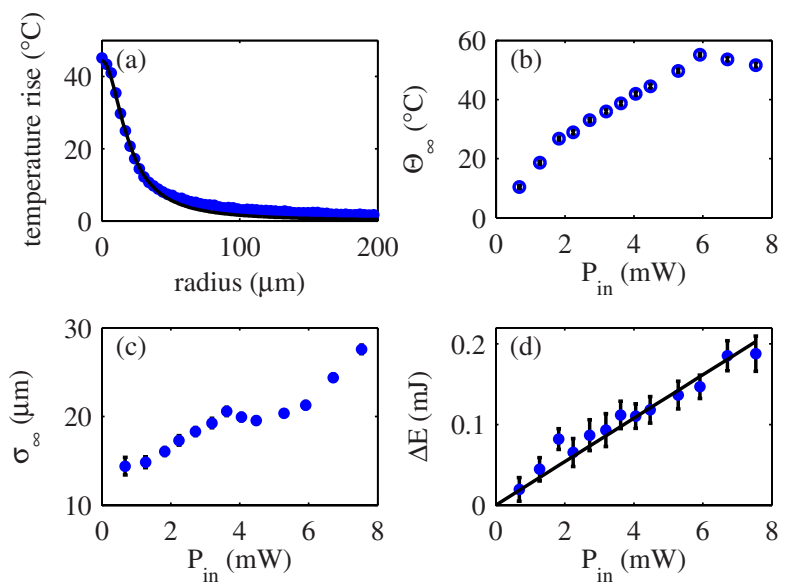

FIG. 7. (Color online) (a) Dots: Radial temperature profile after $0.5 \mathrm{~s}$ of laser heating at $P_{\text {in }}=4.5 \mathrm{~mW}$. Solid line: Lorentzian fit. (b) $\Theta_{\infty}$ as a function of $P_{\text {in. }}$. (c) $\sigma_{\infty}$ as a function of $P_{\text {in. }}$ (d) $\Delta E$ as a function of $P_{\text {in }}$ (dots) and linear fit (solid line). For (b)-(d), the error bars here were calculated as the standard deviation of the data for all the time steps between $0.5<t<1 \mathrm{~s}$. 

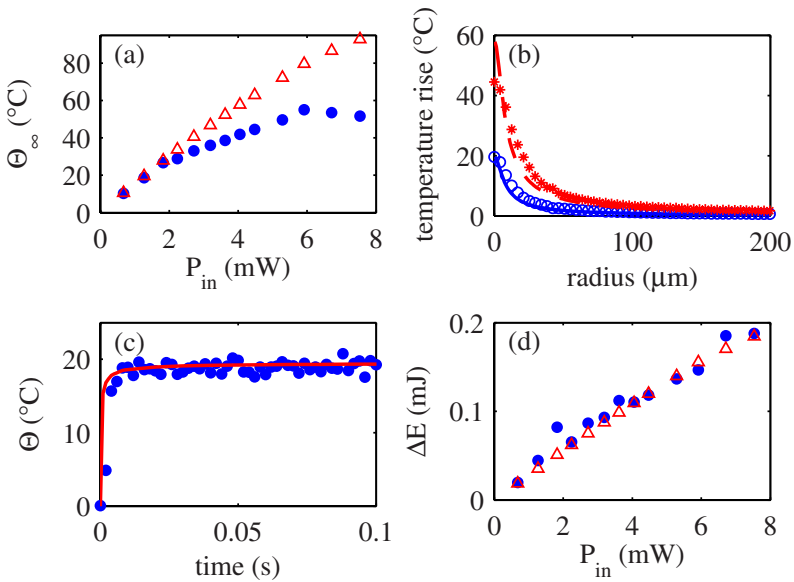

FIG. 8. (Color online) Comparison between the experimental and the numerical results. (a) $\Theta_{\infty}$ as a function of $P_{\text {in }}$. The dots correspond to the measurements and the triangles to the numerical simulations. (b) Experimentally and numerically obtained radial temperature profiles $\bar{T}_{\infty}(r)$ for $P_{\text {in }}=1.3 \mathrm{~mW}$ (circles and solid line) and $P_{\text {in }}=4.1 \mathrm{~mW}$ (stars and dashed line). (c) $\Theta(t)$ for $P_{\text {in }}$ $=1.3 \mathrm{~mW}$. The dots correspond to the experiment and the solid line to the simulation. (d) $\Delta E$ as a function of $P_{\text {in }}$ obtained by the experiments (dots) and by the simulations (triangles).

laser power $P_{\text {in }}[$ Fig. 7(d)], even in cases when the maximum temperature decreases. Indeed, the decrease in the value of the temperature near the laser focus is balanced by an increase in the size of the hot region in such a way that the total energy remains linear with the power. The slope of $\Delta E\left(P_{\text {in }}\right)$ can be interpreted as a measure of the time required to reach a heat flux equilibrium between the injected energy and the heat dissipation into the solid walls, and therefore characterizes the time scale of establishment of the steady state. We measure this time as $\tau=27 \mathrm{~ms}$, in good agreement with the time taken for the temperature gradient to reach its steady value (Fig. 6) and for the temperature profile to reach its final width, as shown in the inset of Fig. 5(b)

\section{Numerical simulations}

The system of Eqs. (1)-(4) was solved numerically using finite-element commercial software (COMSOL, Multiphysics) [24]. The values extracted from the simulations were compared with the experimental results, as shown in Fig. 8. Quantitative agreement is observed for low heating powers $\left(P_{\text {in }}<2 \mathrm{~mW}\right)$ for the maximum temperature increase [Fig. $8(a)]$, for the shape of the radial temperature profile [Fig. $8(\mathrm{~b})]$, and for the evolution of the temperature increase at the laser position $\Theta(t)$ [Fig. 8(c)]. This agreement breaks down at higher powers, with the simulations producing higher temperatures. Moreover, the width of the calculated temperature profiles remains almost constant at about $10 \mu \mathrm{m}$, therefore failing to account for the broadening of the profile that is observed in the experiments. However, the calculation of the total stored energy yields a good agreement between the experiments and simulations for all laser powers [Fig. 8(d)].

The good agreement between the measurements and the numerical simulations at low laser powers suggests that the
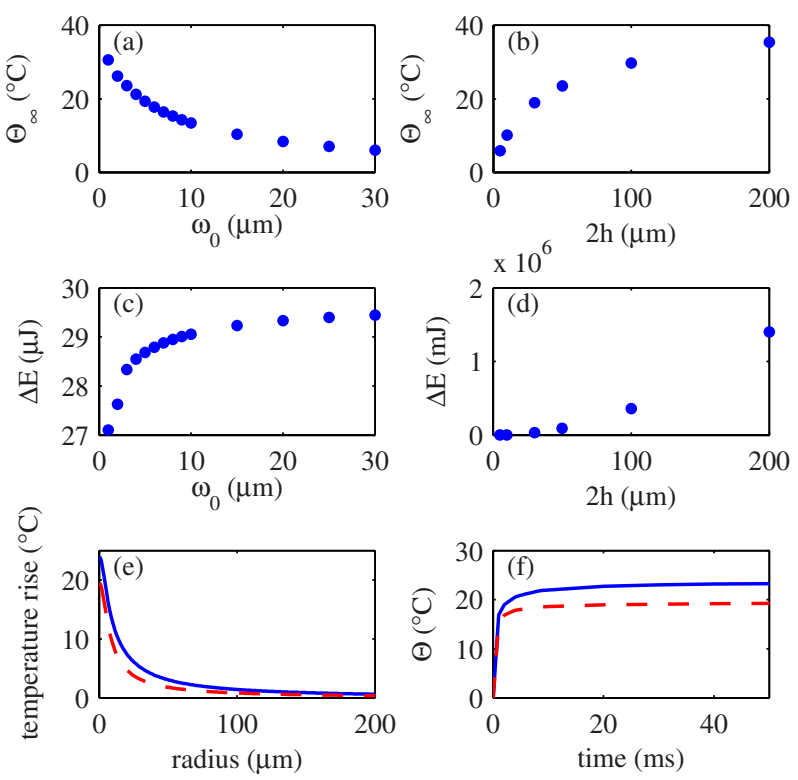

FIG. 9. (Color online) Simulated variations of the main parameters for a laser power $P_{0}=20 \mathrm{~mW}$. (a),(b) $\Theta_{\infty}$ as a function of $\omega_{0}$ and $2 h$, respectively.(c),(d) $\Delta E$ as a function of $\omega_{0}$ and $2 h$, respectively. (e),(f) Comparison between $\bar{T}_{\infty}(r)$ and $\Theta(t)$ for two glass walls (dashed lines) and a combination of one glass and one PDMS wall (solid lines), as in the case of a microchannel.

numerical calculations can be used to describe the heating for different values of the parameters. The values of $\Theta_{\infty}$ and of $\Delta E$ are shown as a function of the laser waist and the depth of the liquid film in Figs. 9(a)-9(d). While the temperature increase is very sensitive to both $\omega_{0}$ and $h$ [Figs. 9(a) and 9(b)], the thermal energy depends only weakly on $\omega_{0}$ [Fig. 9(c)], but strongly on $h$ [Fig. 9(d)].

These results may explain the discrepancy between the measured and simulated temperature profiles, which disagree in the value of $\Theta_{\infty}$ while the total energy shows good agreement. This is coherent with a widening of the laser spot in the experiments, which may be due to the creation of a diverging thermal lens in the lower glass slide as its temperature rises [3]. Such a thermal lens would depend on temperature, which accounts for the increasing discrepancy at high laser powers. Evidence of the existence of this thermal lens can be seen when the sample is viewed with transmitted white light; the location of the laser appears darker than the rest, indicating that the material acts to diverge the light.

Finally, the numerical method is used to calculate the temperature increase in a layer of water enclosed between one glass slide and one polydimethylsiloxane (PDMS) wall, as in the case of a microfluidic device $[9,10]$. The value of the thermal conductivity for PDMS is significantly lower than the value for glass (see Table I), implying that heat will be evacuated less efficiently in the PDMS wall. Consequently, the temperature increase is higher and more extended in this case, as shown in Fig. 9(e). However, the time scale for the increase of $\Theta(t)$, shown in Fig. 9(f), is not significantly modified by the different boundary conditions. 


\section{DISCUSSION}

The establishment of a temperature profile by laser heating takes place over several time scales. The fastest one corresponds to the central laser region reaching its final temperature, which also sets the time required to reach the maximum temperature gradient. This time scale is independent of the laser power and is measured at $\tau_{\Theta}=4.2 \mathrm{~ms}$ in our experiments. Later, the establishment of the width of the Lorentzian profile occurs over a longer time which is associated with the diffusion of the heat into the solid walls; this time will vary with the material properties, although it is also independent of laser power. In our experiments, we measure $\tau=27 \mathrm{~ms}$.

The profile is well described by a Lorentzian curve and the experiments display a nontrivial balance between the width of the hot spot and the height of the temperature peak. These results are not recovered in the numerical simulations, suggesting that the transmission of the laser through the different media is affected by the temperature variations.

Our measurements and simulations can be efficiently used to describe experiments involving laser heating by providing a useful basis for understanding its limitations. Indeed, the steady state profile measured here should still provide a good approximation of the profile expected in the presence of fluid flows, as long as heat diffusion remains faster than advection. This is quantified by the thermal Peclet number Pe $=U L / \chi$, where $U$ is a characteristic velocity and $L$ a characteristic length scale which can be taken as $L=\sigma_{\infty}$, the width of the hot spot. Therefore, by using the values for water and in the conditions discussed in this paper, we find that $\mathrm{Pe}$ $<1$ for characteristic velocities $U<1 \mathrm{~cm} / \mathrm{s}$, which is the case in the previous studies on laser-induced droplet manipulation by thermocapillarity $[9,10,25]$.

Finally, note that our measurements do not take into account the vertical variation of temperature. The numerical solutions show a strong thermal gradient in the $z$ direction, which depends on the material properties of the walls, as was previously shown [17]. These variations should be taken into account if a more precise model of the effect of the thermocapillary flow is needed.

\section{ACKNOWLEDGMENTS}

The authors acknowledge Antigoni Alexandrou for help in the optical measurements. This work was partially funded by the convention X-DGA. M.L.C. was funded by the EADS Corporate Foundation and by MIDEPLAN. E.V. was funded by the CNRS.
[1] K. Fushinobu, L. M. Phinney, and N. C. Tien, Int. J. Heat Mass Transfer 39, 3181 (1996).

[2] G. Da Costa, Appl. Opt. 32, 2143 (1993).

[3] J. P. Gordon, R. C. C. Leite, R. S. Moore, S. P. S. Porto, and J. R. Whinnery, J. Appl. Phys. 36, 3 (1965).

[4] R. Rusconi, L. Isa, and R. Piazza, J. Opt. Soc. Am. B 21, 605 (2004).

[5] H. Reinhardt, P. S. Dittrich, A. Manz, and J. Franzke, Lab Chip 7, 1509 (2007).

[6] L.-C. Ming and W. A. Bassett, Rev. Sci. Instrum. 45, 1115 (1974).

[7] H. Mao, J. R. Arias-Gonzalez, S. B. Smith, I. J. Tinoco, and C. Bustamante, Biophys. J. 89, 1308 (2005).

[8] S. Ebert, K. Travis, B. Lincoln, and J. Guck, Opt. Express 15, 15493 (2007).

[9] C. N. Baroud, J.-P. Delville, F. Gallaire, and R. Wunenburger, Phys. Rev. E 75, 046302 (2007).

[10] C. N. Baroud, M. R. de Saint Vincent, and J.-P. Delville, Lab Chip 7, 1029 (2007).

[11] H. S. Carslaw and J. C. Jaeger, Conduction of Heat in Solids (Clarendon Press, Oxford, 1959).

[12] Y. Liu, D. K. Cheng, G. J. Sonek, M. W. Berns, C. F. Chapman, and B. J. Tromberg, Biophys. J. 68, 2137 (1995).
[13] E. J. G. Peterman, F. Gittes, and C. F. Schmidt, Biophys. J. 84, 1308 (2003).

[14] J. Sakakibara, K. Hishida, and M. Maeda, Int. J. Heat Mass Transfer 40, 3163 (1997).

[15] R. Zondervan, F. Kulzer, H. van der Meer, J. A. J. M. Disselhorst, and M. Orrit, Biophys. J. 90, 2958 (2006).

[16] D. Ross, M. Gaitan, and L. Locascio, Anal. Chem. 73, 4117 (2001).

[17] S. Duhr and D. Braun, Eur. Phys. J. E 15, 277 (2004).

[18] T. Karstens and K. Kobs, J. Phys. Chem. 84, 1871 (1980).

[19] K. G. Casey and E. L. Quitevis, J. Phys. Chem. 92, 6590 (1988).

[20] A. Marcano and O. Urdaneta, Appl. Phys. B: Lasers Opt. 72, 207 (2001).

[21] F. P. Incropera and D. P. De Witt, Fundamentals of Heat and Mass Transfer, 2nd ed. (John Wiley \& Sons, New York, 1985).

[22] S. Duhr and D. Braun, Phys. Rev. Lett. 96, 168301 (2006).

[23] S. Duhr and D. Braun, Phys. Rev. Lett. 97, 038103 (2006).

[24] See EPAPS Document No. E-PLEEE8-78-183812 for a description of the numerical simulation. For more information on EPAPS, see http://www.aip.org/pubservs/epaps.html.

[25] M. R. de Saint Vincent, R. Wunenburger, and J.-P. Delville, Appl. Phys. Lett. 92, 154105 (2008). 